\title{
Ambiguïtés de la réception critique de l'exposition «Canadian Women Artists », Riverside Museum, New York, 1947
}

\section{Édith-Anne Pageot}

Volume 27, numéro 1-2, 2000

URI : https://id.erudit.org/iderudit/1069728ar

DOI : https://doi.org/10.7202/1069728ar

\section{Aller au sommaire du numéro}

\section{Éditeur(s)}

UAAC-AAUC (University Art Association of Canada | Association d'art des universités du Canada)

\section{ISSN}

0315-9906 (imprimé)

1918-4778 (numérique)

\section{Découvrir la revue}

\section{Citer cet article}

Pageot, É.-A. (2000). Ambiguïtés de la réception critique de l'exposition "Canadian Women Artists ", Riverside Museum, New York, 1947. RACAR : Revue d'art canadienne / Canadian Art Review, 27(1-2), 123-134. https://doi.org/10.7202/1069728ar

\section{Résumé de l'article}

In the spring of 1947, the Riverside Museum in New York presented the travelling exhibition, "Canadian Women Artists," bringing together paintings by seventy-two Canadian women, including Emily Carr, Kathleen Daly, Prudence Heward, Anne Savage, Marian D. Scott, Jori Smith and Edna Taçon. The Canadian organizers aimed to make women more visible and to include them within the dominant artistic directions in Canada. The mobilization of women into an international exhibition was without historical precedent. In this respect, the event took on a fundamental importance for the inscription of women in the history of Canadian art. Nevertheless, a heterogeneous collection of works was favoured, and this article intends to show that this orientation weakened the content of the exhibition, favouring questions extraneous to the specificity of the works and to the diffusion of women's art. Only Anne Francis, of the Ottawa Citizen, used the exhibition as an opportunity to denounce the masculine exclusivity of the notion of the creator. Elizabeth Wyn Wood, to whom the introduction of the catalogue had been entrusted, availed herself of the opportunity to valorize the idea of a Canadian artistic temperament which took account of the cultural diversity of the country. The American critic, E.A. Jewell, saw in the exhibition the manifestation of a nationalism that he tried to define by reference to the Group of Seven. For Nettie S. Horch, the director of the Riverside Museum, "Canadian Women Artists" shouldered political aims that overshadowed a singular emphasis on the contribution of women. According to her, the event took up, once more, the efforts of collaboration between nations undertaken by the United States before the war. The feminist purpose, potentially disturbing, was thus reduced by political and ideological considerations.
Tous droits réservés @ UAAC-AAUC (University Art Association of Canada | Association d'art des universités du Canada), 2003
Ce document est protégé par la loi sur le droit d'auteur. L'utilisation des services d'Érudit (y compris la reproduction) est assujettie à sa politique d'utilisation que vous pouvez consulter en ligne.

https://apropos.erudit.org/fr/usagers/politique-dutilisation/ 


\title{
Ambiguités de la réception critique de l'exposition « Canadian Women Artists », Riverside Museum, New York, 1947
}

\author{
ÉDITH-ANNE PAGEOT, UNIVERSITÉ DE MONIRĹAL.
}

\begin{abstract}
In the spring of 1947, the Riverside Museum in New York presented the travelling exhibition. "Canadian Women Artists," bringing together paintings by seventy-two Canadian women, including Emily Carr, Kathleen Daly, Prudence Heward. Anne Savage, Marian D. Scott, Jori Smith and Edna Taçon. The Canadian organizers aimed to make women more visible and to include them within the dominant artistic directions in Canada. The mobilization of women into an international exhibition was without historical precedent. In this respect, the event took on a fundamental importance for the inscription of women in the history of Canadian art. Nevertheless, a heterogeneous collection of works was favoured, and this article intends to show that this orientation weakened the content of the exhibition, favouring questions extraneous to the specificity of the works and to the diffusion of women's art. Only Anne Francis, of the
\end{abstract}

Ottowo Citizen, used the exhibition as an opportunity to denounce the masculine exclusivity of the notion of the creator. Elizabeth Wyn Wood, to whom the introduction of the catalogue had been entrusted, availed herself of the opportunity to valorize the idea of a Canadian artistic temperament which took account of the cultural diversity of the country. The American critic, E.A. Jewell, saw in the exhibition the manifestation of a nationalism that he tried to define by reference to the Group of Seven. For Nettie S. Horch, the director of the Riverside Museum, "Canadian Women Artists" shouldered political aims that overshadowed a singular emphasis on the contribution of women. According to her, the event took up, once more, the efforts of collaboration between nations undertaken by the United States before the war. The feminist purpose, potentially disturbing, was thus reduced by political and ideological considerations.
I du 27 avril au 18 mai 1947 au Riverside Museum' de New York, revient à Edna Breithaupt qui était à l'époque présidente du Arts and Letters Committee of the National Council of Women of Canada. Lors d'une rencontre avec le National Council of Women of the United States, elle avait lancé l'idée de donner aux femmes canadiennes une visibilité sur la scène américaine par le biais d'une exposition: "After a great deal of discussion, Miss Breithaupt's idea of bringing the women of the two countries closer together through mutual cultural interests took root and the American representatives very generously offered to arrange for an exhibition representing women artists across Canada to be held at the Riverside Museum in New York. "2

L'exposition réunissait les oeuvres peintes de soixante-douze artistes canadiennes. ${ }^{3}$ Le catalogue publié, à cette occasion, par le Riverside Museum donne, par ordre alphabétique, la liste des artistes et des oeuvres exposées ainsi que deux courtes présentations signées par les porte-parole des organisatrices américaines et canadiennes. Les tableaux recouvraient les murs de trois salles spacieuses du Riverside Museum, ce qui permettait un accrochage à intervalles réguliers et harmonieux dont le résultat répondait aux attentes des responsables canadiennes. ${ }^{4}$ " $A$ warm wind blew from the North this spring in New York freshening the air and thawing the coldest of art critics. "s Cette opinion exprimée par Pegi Nicol MacLeod n'est qu'un exemple de l'enthousiasme suscité par l'exposition. Si quelques Canadiennes avaient déjà eu l'occasion de participer à des expositions internationales, pensons, par exemple, à la "Women's Art Exhibition" à Détroit, en 1929, une exposition "hors frontières" exclusivement réservée aux Canadiennes n'avait pas de précédent historique. L'exposition du Riverside Museum fut donc une première et fut un succès. ${ }^{6}$ Par ailleurs, le musée avait déjà organisé, en 1939, une exposition internationale consacrée aux artistes féminins d'Europe. ${ }^{7}$ C'est donc huit ans plus tard, conjointement avec le National Council of Women of the United States, que le musée new-yorkais invitait les artistes canadiennes à présenter leur travail au public américain. La réponse des Canadiennes, on l'imagine, fut empressée. Le jury de sélection, formé de Marian Scott, d'Alex Y. Jackson et d'un membre du Conseil, madame R. de Brune Austin, choisit les oeuvres parmi les 536 soumissions reçues par la Eaton Company Fine Arts Gallery. À la demande d'Edna Breithaupt, les oeuvres qui ne furent pas retenues pour New York se virent soumises à un deuxième jury formé, cette fois, de Rody Kenny Courcice, de Kathleen Daly et d'Yvonne McKague Housser, afin qu'elles soient présentées dans une autre exposition, subventionnée, cette fois, par le Council of Women of Toronto, au Wakunda Center. Sous les auspices des différentes branches locales du National Council of Women of Canada, l'exposition de New York voyagea également à travers le pays. Elle séjourna, en effet, dans quelques villes canadiennes: d'abord deux semaines à Toronto, chez T. Eaton Fine Arts Gallery, en septembre 1947, puis à Ottawa, à la Galerie Nationale en octobre de la même année, puis à Kitchener, à London et à Windsor, enfin, en avril 1948, à Montréal, à la galerie d'art d'Eaton.

En général, la réception critique de l'exposition fut positive. Kate Aitken en fit l'éloge: "The collection has good examples of all important trends in Canadian art and is probably the most representative show to go on exhibition outside this country. " $8 \mathrm{Au}$ Québec comme en Ontario, l'exposition récolta une couverture de presse chaleureuse comme en témoignent les commentaires publiés respectivement dans le Montreal Daily Star : All-Woman 
Exhibit Crowned With Success ; variety of subjects attracts interest. ") et le Ottawa Citizen: "It is on the whole an exciting exhibition and a well chosen one. ${ }^{10}$ Autant du côté de la presse francophone qu'anglophone, on fut emballé par l'exposition, une exposition "gonflée " du prestige d'avoir été montrée à New York et d'avoir réussi à retenir l'attention de la critique américaine. Un commentateur anonyme de La Patrie affirmait, par exemple, que: "Un expert en la matière, critique, peintre luimême et curateur d'une galerie d'art, a déclaré aimer $60 \%$ des oeuvres exposées. Il ajouta qu'il avait rarement eu l'occasion d'aimer autant de peintures dans une exposition. "11 Le journaliste attaché au Montreal Daily Star abondait dans le même sens: "Seventy-two Canadian women, two of them dead, startled New Yorkers yesterday - and threatened to startle a good many more in the next three weeks. The New Yorkers were members of the continent's toughest art audience, accustomed to passing judgment on everything from Giotto to Picasso." ${ }^{12}$

Curieusement, cet événement ne retint pas l'attention des historiens d'art. Maria Tippett fut la première à lui consacrer quelques pages dans son livre, By a Lady'13, en 1992. Selon l'historienne, l'exposition avait suscité chez la critique le début d'une remise en question de préjugés qui, à l'époque, situaient l'art des femmes en marge des courants dominants. En effet, nous verrons que l'organisation de cette exposition était une marque tangible d'une certaine mobilisation des femmes engagées professionnellement dans le champ de la peinture. Cependant, on ne saurait établir de lien direct entre la réception critique de l'exposition ex les revendications féministes des années soixante. En scrutant les intentions qui sous-tendent l'événement et les réactions qu'il a suscitées, nous tenterons de démontrer que le propos féministe était en fait largement submergé par des considérations d'ordre idéologique et politique.

\section{L'exposition du Riverside Museum}

Qui exposait et qu'exposait-on dans la "Canadian Women Artists" ? La collection envoyée à New York était constituée de soixante-quatorze toiles, dont la plupart étaient à vendre. On pouvait y voir, entre autres, la peinture naïve de Madeleine Laliberté et de Marie-Cécile Bouchard, La ravaudeuse de Louise Gadbois, L'homme vert de Suzanne Duquet, portrait qui peut être rapproché de la peinture fauve, une étude plus conventionnelle de Marion Long intitulée: The Gay Chintz Smock, une nature morte de Sybil Dobell intitulée: Anémones, trois paysages posthumes de l'ouest canadien d'Emily Carr, Children de Jori Smith, les semi-abstractions d'Edna Taçon et de Marian Scott, La robe blanche de Jeanne Rhéaume, artiste signataire du manifeste Prisme d'Yeux (1948), et une oeuvre de Madeleine Desroches-Noiseux, une automatiste de la première heure. On le constate, l'exposition rassemblait des artistes de différents calibres. Certaines d'entre elles en étaient encore à leurs premières armes, d'autres jouissaient déjà d'une renommée locale et quelques-unes, dont Emily Carr et Lilias Torrance Newton, d'une notoriété nationale. Plusieurs, dont Denyse Gadbois, née en 1921, Ghitta Caiserman, née en 1923, Madeleine Laliberté, née en 1922, et Jeanne Rhéaume, née en 1915, en étaient alors à leurs débuts professionnels. Par conséquent, l'exposition donnait à ces jeunes une visibilité non seulement en terre américaine, mais aussi plus largement sur la scène nationale canadienne. De plus, le voisinage de leur oeuvre avec les paysages plus réputés d'Emily Carr, Gwayasdums d'Sonoqua (vers 1930), par exemple, haussait le prestige de ces artistes moins connues. C'était d'ailleurs l'intention de l'introduction rédigée par Elisabeth Wyn Wood dans le catalogue de l'exposition. Emily Carr ${ }^{14}$ servait en quelque sorte de figure de légitimation pour les autres exposantes. Sa représentation à New York donnait implicitement l'assurance de la qualité de l'exposition. "Some of her [Emily Carr's] best and youngest paintings were made in her last years, years in which she also wrote four distinguished autobiographical books which were "best-sellers" and medal winners in Canada. Her paintings are so respected in Canada that the Vancouver Art Gallery is building a special wing to house them permanently. "15 On trouvait donc, à l'origine du projet, une volonté réelle de promouvoir la carrière de jeunes artistes qui n'avaient pas encore fait leur marque auprès des collectionneurs et le choix des oeuvres correspondait effectivement à ce dessein. ${ }^{16}$ Parmi les artistes dont la carrière était déjà lancée, plusieurs faisaient partie de diverses associations artistiques; c'est le cas notamment de Jori Smith, de Marian Dale Scott, de Louise Gadbois, toutes trois membres fondatrices de la Société d'Art Contemporain et de Kathleen Daly. D'origine ontarienne, Daly était rattachée au Canadian Group of Painters. D'ailleurs, le tableau de Daly envoyé à New York, René, établissait clairement sa filiation avec le Groupe des Sept puisque ce même portrait avait été exposé à la British Empire Exhibition, en 1936. Les Prudence Heward, Mabel Lockerby, Mabel May, Kathleen Morris, Lilias Torrance Newton, Sarah Robertson, Anne Savage et Ethel Seath, quant à elles, avaient toutes été membres de l'éphémère Beaver Hall Group ${ }^{17}$ et jouissaient d'une reconnaissance professionnelle depuis le début des années vingt. Leur participation à l'exposition de 1947 retint l'attention de la plupart des critiques canadiens tout comme l'oeuvre de Marian Dale Scott, Variations on a Theme, Cell and Fossil No. 5, qui fut alors l'artiste la plus prisée ainsi qu'en témoignent les commentaires de Pearl McCarthy dans The Globe and Mail: "Marian Scott's paintings with biological inspiration shine in a class by themselves. ${ }^{18}$ et d'Anne Francis dans l'Ottawa Citizen : "The most interesting painting in the room is, I think, the Variations on a Theme by Marian Scott. ${ }^{19}$ Ces femmes partageaient des préoccupations plastiques qui les rattachaient manifestement à la peinture mo- 
Figure I. Kathleen Morris, After Grand Mass, Berthier-en-haut, 1927, huile sur toile, $61 \times 71 \mathrm{~cm}$, Musée des Beaux-arts de Montréal, don de William James Morrice (crédit photographique: Musée des Beaux-arts de Montréal, Brian Merrett).

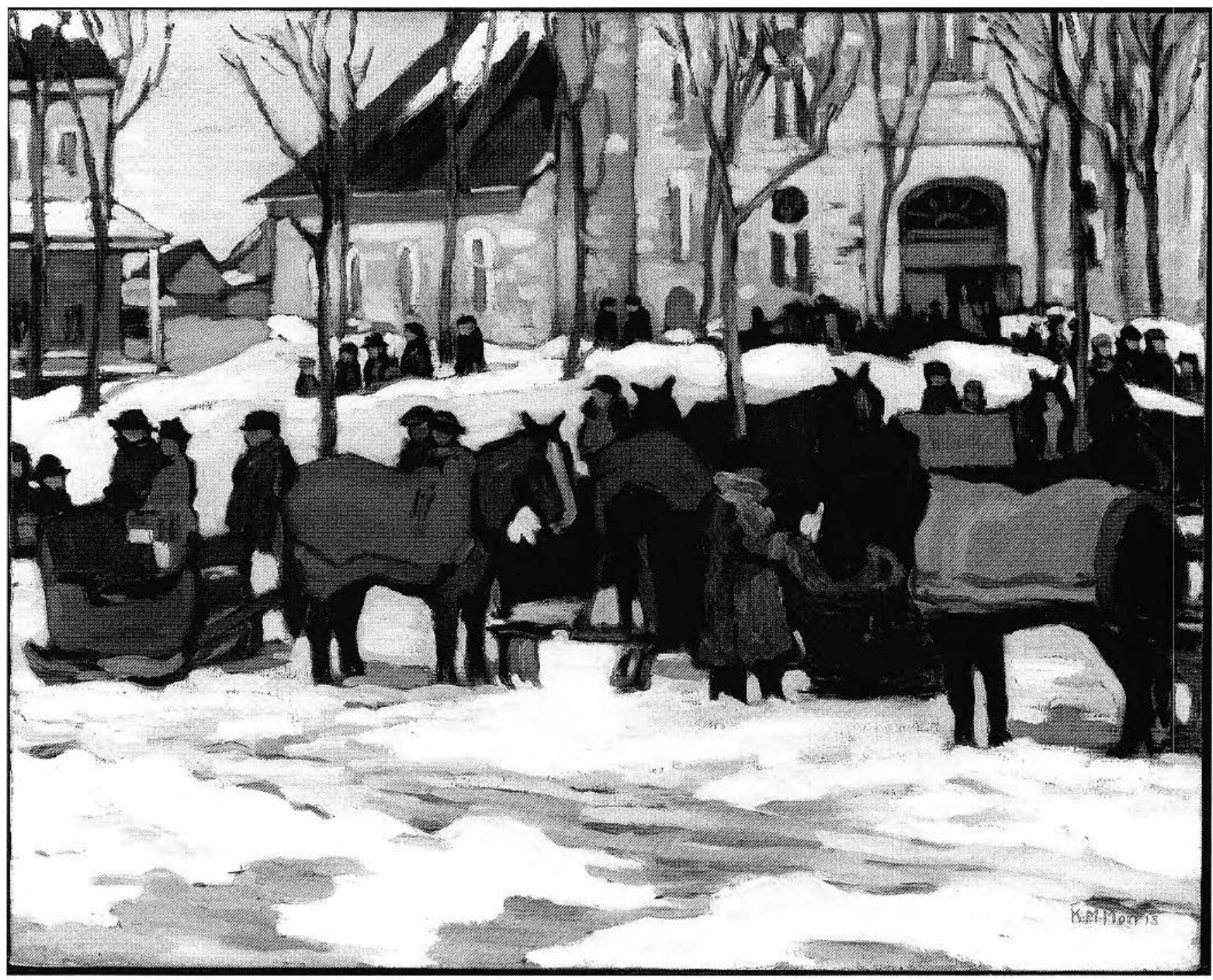

derne des années trente et quarante au Canada. D'ailleurs, After Grand Mass, Berthier-en-haut, 1927 (fig. 1), l'oeuvre de Kathleen Morris présentée à New York, fut l'un des deux seuls tableaux modernes à entrer dans la collection de l'Art Association of Montreal au cours de la même année.

Avec des moyens et à des degrés différents, quelques-unes de ces artistes manifestaient de l'intérêt pour un art public. Plusieurs d'entre elles voulaient démocratiser l'art, ambition qui prenait parfois l'allure d'une pratique plus politiquement engagée comme celle de Paraskeva Clark, Ghitta Caiserman, Peggi Nicol McLeod ou Rose Wiselberg. On sait qu'au cours des années trente, de nombreux artistes canadiens seront tentés de concevoir la pratique artistique comme le lieu d'un engagement social, la crise économique ayant conduit certains à réfléchir sur les rapports entre l'art et la vie. Dans ce contexte, les sujets reliés à la figure humaine seront souvent considérés plus propices à incarner de tels enjeux. ${ }^{20}$ Bien que désinvesti politiquement, le tableau, Variations on a Theme, Cell and Fossil No. 5, de Marian Dale Scott, s'inscrit également dans le sens d'un art public puisqu'il fait partie de sa série Cell and Fossil Forms, 1945-1948, inspirée par ses recherches pour la réalisation de peintures murales du Département de biologie et d'endocrinologie de l'Université McGill, quelques années auparavant. En outre, cette oeuvre est aussi une des rares toiles non figuratives présentées à New York. Aussi minoritaires ou marginales qu'elles aient pu paraître, les oeuvres proches de l'abstraction, comme Metamorphosis d'Edna Taçon, Shelter de Dorothy Henzell Willis ou Rhumbs Un de Madeleine Desroches-Noiseux ont été remarquées par la critique. Cependant, les mentions qui les concernent demeurent très sommaires, il s'agit souvent d'entrefilets. Doit-on attri- 
buer la brièveté des commentaires au caractère déroutant de cette peinture pour une critique d'art habituée à des images plus conventionnelles?21 Dans ce cas, on ne doit pas s'étonner de voir Joséphine Hambleton, critique d'art à l'Ottawa Citizen, qualifier d' "ultra-modernisme » le travail de Marian Scott présenté à New York. "Mrs. Scott belongs to Canada's association of ultra-modern artists, the Contemporary Art Society which was founded in Montreal in 1939. [She] is trying to make some of the current scientific discoveries intelligible in form and colour. She is a pioneer in this type of art in Canada. ${ }^{22}$

Ce tour de salle de l'exposition, bien que non exhaustif, permet tout de même de constater un ensemble assez hétérogène d'approches et de thèmes picturaux. Au fond, on avait envoyé à New York une série d'oeuvres représentant fidèlement les différentes orientations picturales qui coexistaient sur la scène montréalaise et la scène torontoise. Soulignons que la désignation de cette exposition restait discutable ${ }^{23}$, elle regroupait majoritairement des artistes d'origine québécoise et ontarienne, reflétant et valorisant l'importance de Montréal et de Toronto comme centres artistiques au Canada. Kate Aitkens en avait fait la remarque: "Montrealers will be particularly proud of the long list of artists from this city whose paintings were chosen. $»^{24}$ Bref, un tel rassemblement de thématiques et de moyens faisait d'abord et avant tout de l'exposition une rétrospective des oeuvres produites au Canada au cours des deux dernières décennies et montrant les différentes tendances picturales de l'époque. Malgré l'originalité d'une Marian Scott, d'une Émily Carr ou d'une Jeanne Rhéaume pour ne nommer qu'elles, la spécificité de l'exposition ne reposait pas sur une position esthétique précise. La disparité des tendances noyait en quelque sorte les aspects innovateurs ou critiques du travail des unes et des autres. L'originalité de l'exposition résidait davantage dans la mobilisation des artistes féminins. L'événement constituait, en cela, un fait exceptionnel; en effet, on n'avait pas coutume d'exporter une exposition regroupant exclusivement des femmes en terre américaine. Une telle démonstration, "hors frontières", d'un savoir artistique " féminin " était déjà en soi remarquable. L'exposition revêtait donc une importance fondamentale pour l'inscription des femmes dans l'histoire de l'art canadienne.

En dépit de sa nature rétrospective, l'exposition, "Canadian Women Artists " présentait une particularité qui mérite tout de même d'être soulignée : elle accordait à la représentation de la figure humaine une large part qui contrastait avec le paysagisme dominant la scène canadienne à l'époque. En effet, près de la moitié des œuvres étaient se consacrées à ce motif, citons entre autres : My Son, 1941 (fig. 2), de Lilias Torrance Newton, Girl in Blue de Denyse Gadbois, Efa, un portrait d'une nièce de Prudence Heward, The Nunnery d'Anita Elkin. On n'a pas encore mesuré l'importance de la figure humaine dans la peinture canadienne des années trente et du début des années qua-
Figure 2. Lilias Torrance Newton, My Son, 1941, huile sur toile, 77,5 × 57,2 cm, Art Gallery of Ontario, Toronto (crédit photographique: Art Gallery of Ontario).

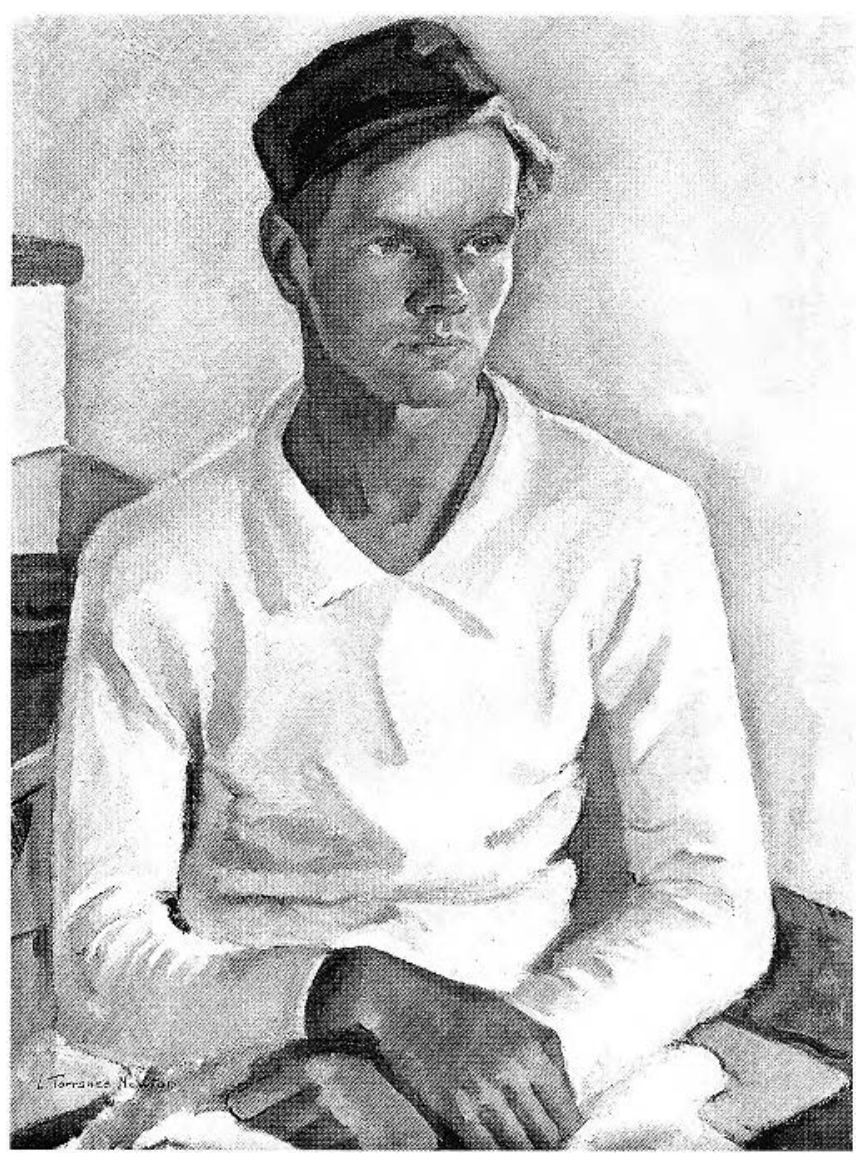

rante ainsi que l'apport des femmes qui furent nombreuses à réinvestir la peinture de la présence humaine. La récurrence de ce motif dans la peinture des femmes, à cette époque, est, à notre avis, une problématique fondamentale et incontournable et on le voit dans la distribution des thèmes de l'exposition. Pourtant, cette particularité a complètement échappé à la critique de l'époque. Un examen des commentaires accompagnant l'exposition, autant dans la revue de presse que dans les textes de présentation, révèle une réalité bien différente des enjeux de l'événement.

\section{Les Canadiennes sur l'échiquier politique américain}

Sous la direction de Nettie S. Horch, présidente du Fine Arts Committee of the National Council of Women of the United States et vice-présidente du Riverside Museum, le musée newyorkais avait organisé plus de vingt expositions regroupant des artistes féminins. ${ }^{25}$ Le programme annuel d'expositions du musée prévoyait une plage strictement réservée aux femmes. Cette assiduité à organiser de telles expositions montre une nette résolution à soutenir leur travail et l'exposition, "Canadian 
Women Artists ", a certainement profité de cette ouverture d'esprit. Pourtant, si cette dernière fait suite en quelque sorte à ces initiatives, elle s'insère également, et peut-être davantage, dans un autre ensemble de manifestations. L'examen de la liste des expositions tenues au Riverside entre 1930 et 1950 montre, d'une part, qu'avant les années quarante, l'idée de présenter des événements consacrés aux femmes provenant de l'extérieur des Etats-Unis ne faisait pas partie de la tradition du musée. Par contre, entre 1940 et 1947, le Riverside importe de l'étranger deux expositions les concernant: l'exposition canadienne qui nous occupe ici et une autre mentionnée en introduction au tout début de la décennie. En effet, du 17 octobre 1939 au 14 janvier 1940, le musée présente au public américain l'exposition, "International Women Painters, Sculptors, Gravers of Australia, Czechoslovakia, France, Greece, Hungary, Italy, Netherlands, Norway, Poland, Switzerland". Or, dans le catalogue de l'exposition, "Canadian Women Artists", Nettie S. Horch faisait explicitement référence à cette exposition internationale de 1939. Ce lien visait manifestement une question identitaire; reste à savoir de quelle identité il s'agissait.

\section{This is a continuation of the pre-war activities of the Fine Arts Committee which has introduced comprehensive exhibitions of women's painting and sculpture from Australia. Czechoslova- kia, France, Greece, Hungary, Italy, Netherlands, Norway, Po- land, and Switzerland at the Riverside Museum in New York City, affording the American public the opportunity to become better acquainted with women's outstanding accomplishments in the Fine Arts Field. 26}

Nettie S. Horch voulait montrer que l'exposition canadienne $s$ 'inscrivaic bel et bien en continuité avec les efforts de collaboration entre les nations déjà entrepris avant la guerre. Cela donne un indice du mandat élargi, déclaré ou non, que s'étaient donné autant la direccion du musée que les instances gouvernementales qui parrainaient les expositions. D'autre part, en consultant la liste des expositions, on remarque également qu'au cours des années quarante, le musée consacrait à l'art étranger une partie de sa programmation: comme les expositions, "Latin American Exhibition of Fine Arts" (1940), "Hiss Philip Hanson of Indonesia" (1941), "Precolumbian Art of Peru in Watercolors" (1941), "New Prints from Latin America " (1942), "The Second Exhibition of the La Tausca Art Exhibition" (1947), cette dernière regroupait des artistes américains de diverses origines ethniques. Fait intéressant, ces expositions proposaient des corpus qui se situaient souvent en marge des pratiques picturales contemporaines, elles présentaient plutôt des grands médiums traditionnels comme les artéfacts archéologiques, la gravure ou l'aquarelle. L'insertion de "Canadian Women Artists" dans cet ensemble d'expositions n'est pas insignifiante. Ces manifestations endossaient, en effet, des visées politiques qui dépassaient la seule mise en valeur de l'apport des femmes, ou de productions peu connues ou moins reconnues de l'art en général. À ce titre, le discours officiel tenu par Nettie S. Horch est révélateur, il s'ouvre sur ces paroles: "Carrying out one of the aims of the National Council of Women of the United States, that of promoting international understanding and good-will [sic], the Fine Arts Committee of the Council has invited the National Council of Women of Canada to organize this exhibition of contemporary women's art of their country. " ${ }^{27}$ Doit-on voir dans cette énonciation d'ouverture des enjeux plus importants que le soutien aux artistes féminins ? Dans le climat politique des années quarante, ceci reviendrait à promouvoir les Etat-Unis comme la nation qui favorise le rapprochement des peuples. C'est ce que laisse entendre la présentation de Nettie S. Horch à l'occasion de l'exposition internationale de 1939:

This International Women's Art Exhibition was conceived with the purpose of cementing the bonds of friendship and to foster greater understanding and good-will [sic] between the women of the United States and Europe. [...] No matter what recent territorial or other changes have taken place in some of the countries represented, the spiritual force of national cultures cannot be destroyed and its aspirations and artistic achievements will live on. ${ }^{28}$

Et c'est également l'approche qu'adopte John D. Morse dans le catalogue de La Tausca:

No one group or faction has dictated style or subject matter.
These pictures represent the main currents in American art
today. Such diversity, with guaranteed freedom of expression for
all, is what makes democracy strong, difficult and exciting. In
view of what happened to German art under Hitler, we may
well be thankful that our painting is still diverse, frequently
strong, sometimes difficult, and always exciting.

C'est un fait qu'à la fin des années quarante, la scène artistique américaine se situe au coeur d'une réorganisation totale de la culture. ${ }^{30}$ Dans ce contexte, la liberté d'expérimentation, étant associée au libéralisme, parait s'opposer aux régimes totalitaires de l'époque. Animés par cet esprit, les organisateurs américains auront voulu montrer leur ouverture en matière de culture. L'exposition, "Canadian Women Artists" remplissait en ce sens une fonction politique auxiliaire, elle offrait l'occasion d'un échange entre deux pays voisins et alliés sur le plan des relations internationales. ${ }^{31}$ En outre, l'accueil de productions venant d'ailleurs et surtout de corpus qui ne se limitaient pas aux courants reconnus ni aux artistes dominants, 
participait à la construction d'une image libérale de la politique culturelle américaine. En s'énonçant au nom de la liberté d'expression et de la coopération internationale, le soutien des instances gouvernementales à la production des femmes favorisait le rayonnement de la démocratie américaine. ${ }^{32}$ Les oeuvres exposées à New York servaient donc d'instrument de propagande politique. Les emprunts de Nettie $S$. Horch à la rhétorique de la guerre froide conféraient aux oeuvres ainsi regroupées une valeur sans doute plus accessible idéologiquement que leur seule qualité esthétique ou la spécificité féminine de l'événement, si bien que les visées politiques du discours officiel amenuisaient le potentiel dérangeant d'une exposition réservées aux femmes.

\section{L'exposition vue par Edward Alden Jewell}

Le critique new-yorkais, Edward Alden Jewell (1888-1947), qui étaic, à l'époque, un collaborateur régulier du New York Times, posa sur l'exposition canadienne un jugement assez favorable. Le ton de Jewell n'était pas étranger à celui de la présentation officielle de Nettie S. Horch. Le critique consacrait à l'événement cinq paragraphes, ce qui est assez long si l'on tient compte de l'envergure relativement modeste de l'exposition. Son article parut deux jours seulement après la date d'ouverture de l'exposition, le 29 avril 1947. Dans ce compte rendu critique, Jewell soulignait la présence de tendances abstraites: il portait à l'attention du futur visiteur la peinture de Hortense Gordon, de Dorochy Henzell Willis, de Madeleine DesrochesNoiseux et d'Edna Taçon. Il n'aurait, semble-t-il, pas remarqué Marian Dale Scott. De plus, il signalait le travail "especially attractive " de Frances-Anne Johnston, de Sybil Dobell, de Denyse Gadbois, de Lilias Torrance Newton, de Julia Crawford et de Kathleen Daly, et il qualifiait de "vigorous " la touche de Paraskeva Clark et de Florence Proctor. Cependant, ces qualifications et classifications demeurent hâtives et n'aboutissent aucunement à une analyse étoffée des approches ou des thèmes. L'auteur se contentait d'énumérer les noms des artistes selon les critères établis plus haut. Au-delà de ces considérations, il voyait plutôt dans cette exposition la manifestation d'un caractère national qui, à l'instar des courants modernes canadiens, se démarquait, disait-il, d'une peinture paysagiste traditionnelle. "One trait that has long characterized the more modern among contemporary Canadian painters is here appropriately marked. This is a trait of stylization, which has many aspects but uniform avoidance of literal transcription from nature. ${ }^{33}$ On l'aura compris, Jewell tentait de définir le "style canadien " par référence au Groupe des Sept. Pour lui, l'intérêt de l'exposition résidait dans une simplification décorative de la forme portant les marques d'une appartenance culturelle, "stylization is marked". Cet intérêt à rechercher et à valoriser un caractère national dans la peincure en général est une des constantes de la pensée de Jewell. On le situe effectivement dans la foulée des critiques et historiens désireux de documenter un art spécifiquement américain. On sait qu'il fut, au cours des années trente et ce jusqu'à la fin de la décennie $^{34}$, un défenseur de l'American Scene Painting. Logique avec lui-même, il tenta de voir également dans la peinture canadienne un profil national: position qui occulte, dans le cas qui nous intéresse, toute réflexion sur d'autres enjeux. Par exemple, Jewell ne faisait pas de commentaires particuliers sur le prétexte féminin de l'exposition. Il rangeait plutôt les artistes dans le lot de la peinture pan-canadienne contemporaine. " [the] Show at Riverside Museum illustrates Contemporary Trends in the Dominion. [...] An effort was made to illustrate various contemporary trends in Canada, and we have the assurance of Elizabeth Wyn Wood of Canadian Arts Council that the exhibition furnishes a fair cross-section. "35 Évitant tout questionnement lié au sexe des exposantes, il se contentait de situer leur travail dans les courants masculins dominants. En réalité, la question de l'insertion des femmes dans le milieu artistique professionnel canadien est un débat qui n'appartient pas au critique américain. Jewell se plaçait davantage du côté de l'observateur à distance appelé à évaluer une autre culture. D'où son intérêt à rechercher, et cela malgré l'éclectisme de l'exposition, un certain style canadien. En vérité, la pluralité des approches picturales retenues renforçait implicitement son argument car elle montrait la capacité de son propre regard critique à saisir l'essentiel dans la diversité! Loin de se pencher sur les enjeux thématiques ou sociaux spécifiques à l'exposition, Jewell a préféré un commentaire qui, tout en confirmant sa propre position en tant que censeur, s'inscrivait logiquement dans la valorisation des dimensions nationales de toute peinture.

\section{«All Canadian and All-Female »}

Le discours officiel des organisatrices canadiennes n'était pas circonspect quant à ses intentions politiques: justice, démocratie et unité nationale s'affichaient comme mots d'ordre. Tout le paragraphe d'introduction du catalogue signé par Elizabeth Wyn Wood est d'ailleurs dévolu à une définition de la nation canadienne:

\footnotetext{
One-half of our population is of British origin, one-third is French-speaking and French-feeling to this day. And we have other large minority groups all of which have brought their gifts to our land and in it are free to speak, live and work according to the traditions of their blood. Time and friendly adjustment have undoubtedly made some changes in the old habits but only in such a way as the pieces of a mosaic are cut to fit the pattern of the whole. The dynamic Canadian slogan is Unity in Diversity. ${ }^{36}$
} 
Toutefois, la pluralité des lieux d'exposition auxquels était destinée la "Canadian Women Artists", au Canada, ne pouvait servir une visée politique comparable à celle des instances américaines. L'exposition fut davantage un lieu de projection et de croisement de diverses perceptions sur le nationalisme et sur l'internationalisme en art. Après tout, il s'agissait, disait-on: "The first all-Canadian and all-female art show $m .{ }^{37} \mathrm{On}$ sait que l'idée d'un art "canadien" revêtait, au cours de la période 1930 1950, des contenus différents et mouvants selon les provinces. Le Groupe des Sept proposait un nationalisme pan-canadien, une vision épurée du paysage qui ignorait les spécificités culturelles composant le Canada. François-Marc Gagnon ${ }^{38}$ a démontré qu'à l'opposé de ce regard décanté, les artistes québécois ont élaboré un régionalisme pictural fondé sur la mise en valeur de leur propre culture. Le plaidoyer d'Elizabeth Wyn Wood pour un nationalisme pan-canadien se situait à la charnière de ces deux pôles. C'était, en fait, une reformulation du nationalisme du Groupe des Sept, une version corrigée qui permettait d'élargir l'art national en incluant l'apport culturel du Québec. De plus, son apologie pour un art canadien sous-estimait les influences européennes sur la peinture concernée. Wyn Wood justifiait les apparentes affinités stylistiques entre les oeuvres canadiennes et les courants européens par une sorte d'intériorisation, assez inexplicable du reste, de la diversité culturelle du pays. Objectant que les Canadiennes n'avaient pas beaucoup étudié outre-mer, elle minimisait toute forme d'apport étranger. Elle souscrivait plutôt à l'idée que l'artiste possède l'inspiration nécessaire au renouvellement de la forme et du sujet. "If a picture seems to have some relationship to French painting, for instance, it is [...] because the blood of old France courses in her modern veins. [...] Our art is rooted in our own soil but has at the same time, we think, some universal qualities. "39 Et elle ajoutait, "In individual works of arts, the diversity of Canadian people is manifest." ${ }^{40}$ Du nationalisme du Groupe des Sept à celui de Wyn Wood, la fiction s'était déplacée de la terre vierge, frustre et imposante, au corps du créateur entendu comme le lieu où s'incarnaient mystérieusement cette nature impénétrable nourrie du terreau des cultures ancestrales!

S'appuyant sur la présentation de Wyn Wood, les critiques montréalaise et torontoise insistaient, elles aussi, sur la reconnaissance et la mise en valeur d'un certain tempérament canadien. On magnifiait les mérites de cette exposition qui était la preuve de l'existence d'un art national, affranchi d'influences extérieures et indépendant d'esprit. Un critique du Montreal Daily Star avouait: "What surprised most New Yorkers was that Canadian painting, in whatever style, was obviously and completely Canadian in form as well as subject matter. " ${ }^{41}$ Pearl McCarthy abondait dans le même sens: "Long observation of Canadian work leaves one able to agree with what Elizabeth Wyn Wood said in the foreword of the New York catalogue [...] that they [the pictures] represent Canadians going through the same mental process. " 42

Il s'agit là de questions tout à faic typiques de l'époque; on revendiquait l'expression de la subjectivité en peinture tout en valorisant et en perpétuant l'idée que les artistes étaient des êtres inspirés et que le génic artistique existait. Cependant, dans ce cas-ci, le génie était féminin. La définition du génie artistique canadien servait donc tacitement à situer la production des femmes et à préciser leur statut sur la scène canadienne.

En dépit d'une caractérisation un peu forcée de la spécificité nationale, la présentation de Wyn Wood montre à quel point il paraissait nécessaire, dans les années quarante, d'affirmer la compétence et le professionnalisme des femmes. L'exposition était donc une occasion de confronter un certain nombre de questions débattues à l'époque au problème de définition que représentait un "art féminin". L'exposition, "Canadian Women Artists " devenait-elle alors un avant-courricr des expositions féministes des années soixante ? L'exposition établissait les conditions nécessaires à un certain questionnement sur la place et la spécificité des femmes. Il s'agissait de montrer leur apport aux principales tendances artistiques qui coexistaient au Canada au cours des années trente et quarante. Et la critique a effectivement insisté sur la capacité de l'exposition à le faire : "Certainly, the paintings show competence because the established Canadian women painters are there. ${ }^{43}$ De plus, elle coïncidait dans le temps avec d'autres expositions réservées exclusivement aux femmes. L'initiative new-yorkaise est, en effet, la deuxième d'au moins quatre autres manifestations semblables tenues entre 1947 et 1950. La même année, en 1947, le Musée de la Province de Québec organisait l'exposition, Fémina ${ }^{44}$, une année plus tard, la Eaton Art Gallery montrait au public montréalais une exposition qui s'intitulait: Femmes peintres au Canada ${ }^{45}$, suivie, au printemps 1949, de "Canadian Women Painters" ${ }^{46}$, à la West End Art Gallery, et de la AAM, en 1950, avec son " Group Show of Women Painters $\cdots{ }^{47}$

Une telle diffusion à l'intérieur d'une période aussi courte ne s'était encore jamais vue à Québec ni à Montréal, car bien qu'à partir des années vingt, les femmes professionnelles exposaient aux côtés de leurs collègues masculins, au Salon du Printemps de l'Art Association, au salon de l'Académie Royale, dans les galeries, dans les expositions privées et, parfois, dans les librairies. Les expositions de groupe réservées exclusivement aux artistes professionnelles demeuraient rares $^{48}$ et avaient peu à voir avec la moindre revendication articulée. Il est vrai que la Women Art's Society organisait annuellement une présentation d'œuvres issues de la Society's Studio Group. Il s'agissait essentiellement d'expositions de travaux d'amateures auxquelles on ajoutait quelques œuvres d'artistes professionnelles dans le but avoué "to raise the standards of the whole show $"{ }^{49} \mathrm{Si}$ on écarte les expositions de la Women Art's Society ${ }^{50}$, la liste des exposi- 
tions de groupe consacrées exclusivement aux artistes féminins professionnels entre 1930 et 1945 demeure considérablement restreinte $^{51}$. Leur augmentation soudaine entre 1947 et 1950 indiquerait non seulement l'existence d'une pratique plus largement répandue mais aussi un besoin d'organiser un réseau de diffusion pour les femmes; la tentative de les insérer dans l'histoire de l'art par des expositions remonterait donc aux années quarante et cette situation transformait nécessairement la configuration de la scène artistique canadienne. Conséquemment, on est en droit de se demander comment la critique a relevé le défi que représentait l'exposition, "Canadian Women Artists "? Kate Aitken du Standard ${ }^{52}$ passa complètement sous silence la spécificité féminine de l'événement. Il s'agissait pour elle d'affirmer le caractère canadien de l'exposition. Pearl McCarthy, dans The Globe and Mail, tentait, quant à elle, de situer la peinture des femmes au même niveau que celle des hommes:

\section{No feminist prejudice need be suspected in the statement that it is one of the strongest Canadian collections ever exported. for men have been among those who have spoken of having to go back to the Wembley exhibit, in the zenith of the Group of Seven leadership, to find a Canadian representation of equal verve. This does not infer that women painters of the moment are better than men. It does mean that this exhibition was well managed and that there were good women painters available. ${ }^{53}$}

De toute évidence, elle tentait de prouver l'égalité des compétences entre les artistes masculins et féminins. Nonobstant l'affirmation qu'elle faisait du professionnalisme des femmes, McCarthy recourait néanmoins au jugement des hommes pour le démontrer. Ceci indique à quel point la voix masculine était idéologiquement garante du sérieux et de l'importance d'une manifestation de ce genre. De plus, en évoquant le rôle traditionnel d'organisatrice joué par les femmes dans les milieux artistiques canadiens, l'auteure atténuait le contenu " agitateur " de ses remarques. Le lecteur pouvait ainsi facilement rabattre le succès de l'exposition sur le travail de soutien plutôt que sur le travail créateur des femmes impliquées dans l'événement. Anne Francis, critique à l'Ottawa Citizen, avait vu l'exposition à la Galerie Nationale, à Ottawa, en octobre 1947. Elle était, sans aucun doute, la plus engagée de tous les commentateurs de l'exposition. Elle adoptait d'ailleurs un ton plus agressif et son argumentation était mieux articulée que celle de McCarthy, par exemple. Elle demeura la seule à se pencher ouvertement sur les enjeux féministes de l'exposition et à sortir des thèses nationalistes centrées autour de la pratique du Groupe des Sept. Francis désirait montrer qu'on retrouvait parmi les femmes des peintres qui avaient véritablement acquis une maturité artistique, leur travail n'étant pas la fade copie de celui des hommes. Elle ouvrait la voie à la remise en question de valeurs reçues.
It seems inevitable that the hand of the Group of Seven should still guide the brush of our landscape painters. This show is no exception. However the influence is certainly less compelling than it was ten or even five years ago when every spring exhibition was crammed with near Thomsons and Jacksons (not so very near either). (...) If they are good and really have something to say they go on to develop their own style in due course. ${ }^{54}$

En écrivant cela, elle dissociait le talent arcistique de toute détermination sexuelle et affirmait l'autonomie et l'originalité des peintres féminins les plus doués. Sur un ton beaucoup plus provocateur cette fois, elle questionnait aussi la pertinence de classer les artistes selon leur sexe. "If you want to make a professional woman mad tell her that she writes well or is a good doctor - "for a woman". Obviously sex should have nothing to do with the evaluation of ability or talent. The exhibition of 57 paintings by women artists at the National Gallery is a happy example of this truth. "S5 Ses remarques visaient les préjugés rattachant l'art des femmes, et la féminité en général, à des capacités plus réduites; elles refusaient à la série de tableaux envoyée à New York une spécificité féminine telle qu'on pouvait la concevoir à l'époque et telle qu'on la retrouve parfois chez quelques commentateurs anonymes. C'est le cas notamment de l'article paru dans le Montreal Daily Star: "The Americans apparently were startled because they didn't expect much from Canadian artists, particularly women. "56 et d'un autre publié dans La Patrie : "Le public américain est stupéfait, tout d'abord, parce qu'il ne s'attendait nullement à trouver autant d'artistes chez nous et surtout autant de femmes peintres. "57

Rappelons que la peinture canadienne produite par les femmes jusqu'au début du XXe siècle demeurait le plus souvent sans conséquences économiques, elle était rarement destinée au marché privé ou à la décoration des édifices publics et religieux. Les arts dits féminins se retrouvaient dans la sphère limitée du loisir, de l'activité culturelle et des bonnes manières, bref dans l'espace domestique élargi. ${ }^{58}$ À la fin des années quarante, cette conception domine encore la pensée de bon nombre d'intellectuels, tel Marcel Clément lorsqu'il présentait au public québécois l'enseignement du pape Pie XII sur la femme et sur son rôle dans la société:

Ce qui donnera une âme au tout, c'est la main et l'art de la femme, qui permettront à l'épouse de rendre attrayants tous les coins du foyer. [...] Dieu a donné à la femme plus qu'à l'homme, avec le sens de la grâce et de la beauté, le don de rendre aimables et familières les choses les plus simples, et cela précisément parce que, créée semblable à l'homme pour former avec lui une famille, elle est faite pour répandre le charme et la douceur au foyer de son mari et y assurer à deux une vie féconde et florissante. ${ }^{59}$ 
Le passage évoque évidemment un contexte beaucoup plus large que la seule réception critique de l'exposition de New York, mais il reste que ce genre de commentaire dresse un portrait général d'une certaine représentation de la féminité ainsi que des qualités et des rôles qui s'y rattachaient à l'époque. Or, la peinture dite féminine se trouvait inévitablement liée à cette image; elle était d'ailleurs souvent comprise comme une mise en forme et une incarnation du féminin. ${ }^{60}$ On retrouve cette perception chcz Charles Doyon lorsqu'il rédige la critique des expositions de groupe réservées aux femmes, contemporaines à la "Canadian Women Artists". Sous sa plume, la "peinture féminine " déborde de "délicatesse ", de "douceur " et de " tendresse ". ${ }^{61}$ Cette conception se retrouve aussi dans la réception critique de l'exposition, chez Renée Normand ${ }^{62}$ notamment, à qui le journal Le Canada avait confié la tâche de commenter l'exposition. Elle voyait chez Bess Harris, une artiste de Vancouver, ainsi que chez la montréalaise M. R. Holland et chez Carmen Sherbeck d'Edmonton, "élégance ", "facilité " et "charme ». En fait, ces réactions n'avaient rien de nouveau dans les milieux artistiques canadiens. Charles Doyon tout comme Renée Normand reconduisaient les atcitudes et les stéréorypes formulés quinze ans plus tôt par Henri Girard à l'occasion de l'une des rares, et, sans doute, une des premières expositions de groupe réunissant à la galerie Eaton, à Montréal, des artistes féminins professionnels. Les commentaires de Girard montrent que la figure de l'artiste et l'identité féminine étaient encore difficiles à concilier:

L'élite humaine, avide de perfection, attache ses regards aux sommets éternels où le génie a de ses poings d'acier forgé l'Art. [...] Les vrais amateurs cependant, ne méprisent pas un art plus modeste, langage harmonieux des âmes de bonne volonté. C'est la grande route du bien dire, chemin fleuri qui côtoie les abîmes du néant, art délicat et discret qui parle à mi-voix. Poète ou peintre la femme artiste y trouve un mode d'expression mesuré qui convicnt à sa nature. Ici règne je ne sais quoi où l'éternelle Eve affirme sa propre puissance, ombrée de ses faiblesses. ${ }^{63}$

On cherche à faire reconnaitre dans la production artistique des femmes une féminité présumée naturelle à laquelle correspondent également un certain nombre de valeurs: délicatesse, douceur, tendresse, mollesse, disponibilité, etc. En convoquant ainsi une spécificité fantasmée on rangeait implicitement la "peinture féminine" dans des catégories secondaires. On voyait dans les œuvres des femmes quelque chose d'autre, un " nouveau genre ", selon Doyon. Ces expressions indiquent clairement le statut marginal dans lequel on plaçait les femmes:

Grâce à l'initiative de Miss Millman, propriétaire du West End Gallery, les amateurs de peinture auront l'occasion durant les premières semaines d'avril, de se familiariser avec la peinture féminine à Montréal. [...] Parmi ces dernières quelques-unes sont avantageusement connues des amateurs de peinture : Émilie Carr, Louise Gadbois, Prudence Heward, décédée récemment, Anne Savage, Marion Scott [sic] et Jori Smith, ce qui fait que dans leurs meilleurs œuvres, elles peuvent quelquefois se comparer avec des peintres masculins $[\ldots]^{64}$

Par contre, les tableaux les mieux réussis, ceux, justement, qu'on pouvait comparer aux oeuvres des collègues masculins, étaient caractérisés par des qualificatifs culturellement attribués à la virilité, telles que vigueur, force, fermeté, solidité et robusresse. ${ }^{65}$ Ainsi, Renée Normand conférait à Suzanne Duquer des traits de ce genre. "Un des meilleurs peintres que nous ayons, peint avec une force étonnante, dessine avec une sûreté de ligne qui ne faiblit jamais.» ${ }^{66}$ Ces remarques faisaient, encore une fois, écho à celles d'Henri Girard lorsqu'il jugeait, dans l'article cité plus haut, le travail de Sarah Robertson et celui de Jori Smith. Devant les oeuvres jugées réussies et produites par des femmes, on préférait refouler, sans pour autant nier son existence, cette féminité qui à d'autres moments était tant recherchée. Par exemple, Renée Normand convoquait à nouveau la nature féminine lorsqu'il s'agissait de justifier l'iniquité de la représentation proportionnelle des femmes et des hommes dans les collections canadiennes. Selon elle, la disposition des femmes à la sincérité et au détachement matériel expliquait les difficultés rencontrées par celles-ci dans la promotion de leur travail:

De l'ensemble de l'exposition se dégageait beaucoup de sincérité. La femme-artisce est en butte aux mêmes difficultés quc l'homme, avec ceci en plus que son talent passe trop souvent inaperçu, surtout s'il refuse de se faire à lui-même une publicité tapageuse. Les peintres dont j'ai parlé plus particulièrement semblent se soucier bien peu de la "gloire", ce qui leur permet d'être infiniment plus honnêtes dans leur art. Elles peignent comme elles l'entendent, sans considération pour l'acheteur possible ou les exigences mondaines; et quand sur soixante et quelques toiles on peut trouver six qui ont du mérite, on s'incline bien bas: le fait est unique. ${ }^{67}$

Si Renée Normand tentaic de dégager la production des artistes professionnelles de certains préjugés, comme celui de la complaisance mondaine souvent attribuée aux femmes bourgeoises impliquées dans le champ des arts, elle évitait toutes les questions qui auraient pu conduire à des revendications d'égalité face aux moyens de diffusion. La nature féminine, honnête et sincère, portait à elle seule le fardeau d'une situation inacceptable mais en quelque sorte prédestinée! Bref, l'usage qu'elle faisait 
d'une terminologie reliée au sexe pour désigner le travail des exposantes consolidait indirectement la séparation entre le féminin et le masculin tout en confirmant la nature masculine du "génie » artistique.

Finalement, l'exposition "Canadian Women Artists » représentait véritablement un défi à la critique canadienne. Défi qu'on aura négocié de deux façons: en convoquant la féminité, ce qui offrait l'avantage de ranger la " peinture féminine " dans une catégorie à part et secondaire ou, pour ce qui concerne les oeuvres jugées particulièrement réussies et comparables à celles des hommes, en refoulant le féminin, ce qui évitait de questionner les préjugés les mieux établis. L'exposition aura été comprise par quelques-uns comme une manifestation d'un genre particulier et peu menaçant pour le canon. La manifestation en soi n'avait rien de rebelle et n'ébranlait pas les certitudes de l'histoire de l'art canadienne. Elle avait malgré tout engendré chez Anne Francis et, dans une moindre mesure, chez Pearl McCarthy, des questionnements sur l'exclusivité masculine de la notion de grand créateur. La réaction critique d'Anne Francis et celle de Pearl McCarthy montrent que l'exposition de New York était aussi un lieu d'ancrage et de ventilation de la conscience collective à l'égard des condirions d'exposition de l'arr des femmes au Canada. Quant aux intentions des organisatrices, elles visaient à donner aux femmes une visibilité, à encourager les jeunes et surtout à inclure les femmes dans les courants picturaux dominants de l'art canadien. On avait donc privilégié un ensemble disparate de propositions esthétiques. La sélection des oeuvres évitait de trouver une spécificité de l'art des femmes; voulant plutôt "libérer" les femmes de la "peinture féminine". En évitant la focalisation sur un thème ou sur une démarche plus précise, le contenu de l'exposition s'en trouvait affaibli et favorisait les questions étrangères aux œuvres. Les Américains trouvèrent là une occasion d'afficher certaines positions liées au contexte politique de la guerre froide et, du côté canadien, l'exposition offrait une autre occasion pour débattre la question du nationalisme en art.

\section{Notes}

1 I.e Riverside Museum était situé au 310 Riverside, au coin de la $103^{e}$ Rue, à Manhattan, au nord-ouest de Central Park. Ce musée a été fondé par Nicolas Roerich. Aujourd'hui fermé, il était à l'époque aménagé à l'étage inférieur du Master Institute of United Arts.

2 Canada Foundation Papers, "Canadian Arts Council ". NAC, MG 281179 , vol. 26, p. 3.

3 Marion Aronson, Marie Cécile Bouchard, Mary Bouchard, Ray Burridge, Portia Ruth Butt, Ghitta Caiserman, Viola Campbell, Emily Carr, Nan Lawson Cheney, Paraskeva Clark, Ann Clendening, Rody Kenny Courtice, Julia Crawford, Ethel Curry, Kathleen Daly, Gwen Dawson, Madeleine Desroches-Noiseux, Sybil Dobell, Bernice Drummond, Suzanne Duquet, Anita Elkin, Gladys Ewan,
Marguerite Fainmel, Mary Fergie, Mary Filer, Helen Fitzgerald, Emme Frankenberg, Denyse Gadbois, Louise Gadbois, Hortence Gordon, Bess Harris, B. Cogil Haworth, Prudence Heward, Hulda Hill, M.R. Holland, Yvonne McKague Housser, Melisande Irvine, Maud Jemmett, Frances-Anne Johnston, Madeleine Laliberté, Agnès Lefort, Mabel Lockerby, Marion Long, Virginia Luz, Peggi Nichol MacLeod, Betty Maw, Mabel May, Doris MacCarthy, Isabel McLaughlin, Margaret Mclaughlin, Marjorie Meredith, Kathleen Morris, Frances Neil, Lilias Torrance Newton, Françoise Picard, Aline Piché, Florence Proctor, Jeanne Rhéaume, Sarah M. Robertson, Anne Savage, Marian D. Scott, Ethel Seath, Irène Sénécal, M. Carmen Sherbeck, Jori Smith, Dorothy Stevens, Helen Stevens, Edna Taçon, Agnès Warren, Dorothy Henzell Willis et Rose Wilselberg. Catalogue de l'exposition.

4 "In New York the exhibition was beautifully hung. There were lighted galleries, the paintings were generously spaced and everyone to whom I spoke was more than pleased with the representation of the Exhibition at the Riverside Museum. "Yvonne McKague Housser, Canada Foundation Papers, Canadian Arts Council, Galeric Nationale du Canada, MG 281 179, vol. 26.

5 Propos de Pegi Nicol MacLeod cités: Anonyme, "First Showing in Canada! "Canadian Women Painters" as Presented in New York, April 1947 ", Toronto Daily Star, 3 septembre 1947.

6 Selon le journaliste anonyme du Montreal Daily Star, le succès de l'exposition avait valu à Françoise Picard, remarquée semble-t-il par Robert Bonfils, une bourse de l'Ecole des beaux-arts de Montréal afin d'étudier à Paris. "The show, incidentally, has produced a concrete result: Studio by Françoise Picard, came to the attention of Robert Bonfils of the Ecole des Beaux-arts, with the result that Mlle Picard is going to Paris on a scholarship". "N.Y. Art Show Features Works of Canadians", Montreal Daily Star, 29 avril 1947. Robert Bonfils était un parisien, professeur, peintre et décorateur. On ne sait pas s'il avait vu l'exposition du Riverside Museum. À la même date il donnait une causerie intitulée "Artistes et artisans dans la nation " devant les membres de l'Association des techniciennes laïques en sciences ménagères à Montréal. (Anonyme, "Les efforts artistiques du Canada loués par un Français ", La Patrie, 29 avril 1947.) Quoiqu'il en soit, l'intérêt manifesté par Bonfils pour la peinture de l'artiste canadienne ajoutait aux yeux de la critique montréalaise du prestige à l'exposition de New York.

7 Du 17 octobre 1939 au 14 janvier 1940. Pour le titre exact de l'exposition voir la section de cet article intitulée: "Les Canadiennes sur l'échiquier américain."

8 Kate Aitken, "Montreal Women's Art Chosen for NY Exhibit ", The Standard, 29 mars 1947.

9 Anonyme, Montreal Daily Star, art. cit.

10 Anne Francis, "Work of Women Artists ", Ottawa Citizen, 10 octobre 1947.

11 Anonyme, "Des Canadiennes ont conquis New York ", La Patrie, 29 avril 1947.

12 Anonyme, Montreal Daily Star, art. cit.

13 Maria Tippett, By a Lady, Celebrating Three Centuries of Art by Canadian Women, Viking, Toronto, 1992, p. 109-111. 
14 Tel qu'indiqué dans le catalogue, les oeuvres d'Emily Carr présentées à l'exposition étaient: In the Woods, Trees, Guyasdoms d'Sonoagua [sic]. Certains titres du catalogue comportent des erreurs, on ne donne jamais la date, de plus les collections auxquelles appartiennent les oeuvres ne sont pas toujours indiquées.

15 Elizabeth Wyn Wood, Canadian Women Artists, catalogue de l'exposition, New York, Riverside Museum, 1947, n. p.

16 Canada Foundation Papers, Yvonne McKague Housser, Galerie nationale du Canada, MG 281 179, vol. 26, p. 2.

17 Il ne manquait qu' Emily Coonan et Nora F. Collyer.

18 Pearl McCarthy, The Globe and Mail, 4 septembre 1947.

19 Anne Francis, art. cit.

20 Voir à ce sujet les positions de François-Marc Gagnon, "La peinture des années trente au Québec ", Annales d'histoire de l'art canadien/The Journal of Canadian Art History, vol. 3, $n^{\text {os }} 1$ et 2, automne 1976, p. 2-20, et d'Esther Trépanier, Peintres juifs et modernité, Montréal 1930-1945, Montréal, Centre Saidye Bronfman, 1987.

21 François-Marc Gagnon, "Le sens du mot "abstraction" dans la critique d'art et les déclarations de peintres des années quarante au Québec ", Yvan Lamonde et Esther Trépanier, L'avènement de la modernité culturelle au Québec, Montréal, Institut de recherche québécois sur la culcure, 1986, p. 114-138.

22 Josephine Hambleton, "Cell and Crystal in Modern Art ", Ottawa Citizen, 4 octobre 1947.

23 Vingt-neuf des exposantes résidaient au Québec et trente-deux en Ontario, ce qui laissait à la Colombie-Britannique six participantes, tandis que la province de la Saskatchewan était représentée par une seule artiste tout comme l'Alberta, la Nouvelle-Écosse, le Nouveau-Brunswick et le Manitoba .

24 Kate Aitken, art. cit.

25 En 1947, le Riverside Museum en était à sa 23ième exposition annuelle de la New York Society of Women Artists, tenue du 3 au 16 novembre 1947.

26 Netrie S. Horch, op. cit.

27 Ibid.

28 Nettie S. Horch, International Women Painters-Sculptors-Gravers, catalogue d'exposition, New York, Riverside Museum, du 17 octobre 1939 au 14 janvier 1940. Présentée à ce musée, l'exposition était organisée par le National Council of Women of the United States et parrainée par l'International Council of Women.

29 John I). Morse, La Tausca Art Exhibition, catalogue d'exposition, New York, Riverside Museum, du 24 janvier au 8 février 1947. Au cours de l'année 1947, cette exposition a circulé dans différents états américains sous les auspices de l'American Federation of Arts. Son itinéraire étaic le suivant: le Riverside Museum à New York, New York, du 24 janvier au 8 février; l'Oklahoma Art Center, Oklahoma City, du 28 février au 16 mars; le Laycon Art Center à Milwaukee, Wisconsin, du 31 mars au 20 avril; le Isaac Delgado Museum of Art à New Orleans, Louisiane, du 4 mai au 25 mai; le J. B. Speed Memorial Museum à Louisville, Kentucky, du 8 juin au 29 juin; le Museum of Fine Arts à Houston, Texas, du 13 juillet au 3 août; le Detroit Institute of Arts, Detroit, Michigan, du 17 août au 7 septembre; le Baltimore Museum of Arts, Baltimore, Maryland, du 18 septembre au 8 octobrc; le Walker Art Center à
Minncapolis, Minnesota, du 26 octobre au 16 novembrc; le Cleveland Museum of Art, Cleveland, Ohio, du 2 décembre au 4 janvier.

30 Serge Guilbault, "The Frightening Freedom of the Brush: The Boston Institute of Contemporary Art and Modern Art ", Art Apart, Manchester et New York, Manchester University Press, 1994.

31 Au sortir du deuxième conflit mondial, les relations internationales connaissent un développement sans précédent historiquc. L'objectif manifeste des grandes organisations nouvellement formées (les accords du GATT de 1947, de l'OTAN, la création de l'ONU émanant de cctte même volonté politique) est, sans contredit, la coopération internationale. Cependant, à l'instar de la Société des Nations, ces organisations vouées au maintien de l'ordre international issu de la seconde guerre mondiale visent la préservation de la coalition victorieuse des alliés comme le révèle l'attribution d'un siège permanent et d'un droit de vcto au sein du Conseil de Sécurité aux cinq principales puissances victorieuses. En ce sens, le développement des relations internationales s'inscrit dans le contexte plus large de la guerre froide. Voir les analyses de Harold K. Jacobson, Networks of Interdependence. International Organizations and the Global Political System, New York, Alfred A. Knopf, 1979, Michel Velleray, L'organisation mondiale, Paris, Librairie Armand Colin, 1972, et André Fontaine, Histoire de la guerre froide: De la Révolution d'octobre à la guerre de Corée (1917-1950), Tome I, Paris, Fayard, 1967.

32 Pour une discussion théorique et critique des rapports entre la valeur politique et la valeur esthétique des pratiques d'expositions depuis la seconde guerre mondiale dans les sociétés occidentales, voir Rainer Rochlitz, Subversion et subvention. Art contemporain et argumentation esthétique, Paris, Gallimard, 1994.

33 Edward Alden Jewell, "Canadian Women Offer Art Display", New York Times, 29 avril 1947.

34 À la fin de 1939, l'American Scene Painting dont sont issus le régionalisme pictural et le réalisme social, faisait ni plus ni moins déjà partie de l'histoire; l'ouvrage de Jewell, publié en 1939, marque en quelque sorte la fin de ce mouvement pictural. E. A. Jewell, Have We an American Art?, Ncw York, Longmans, Green Company, 1939. Il est également l'auteur d'Americans, New York, Alfred A. Knopf, 1930, entre autres.

35 Edward Alden Jewell, art. cit.

36 Elisabeth Wyn Wood, art. cit.

37 Anonyme, Montreal Daily Star, art. cit.

38 François-Marc Gagnon, "La peinture des années trente au Québec ", art. cit.

39 Elisabeth Wyn Wood, art. cit.

40 Ibid.

41 Anonyme, Montreal Daily Star, art. cit.

42 Pearl McCarthy, art. cit.

43 Anne Francis, art. cit.

44 Fémina, 10 février au 16 mars 1947, Musée de la Province de Québec. L'exposition réunissait: Simone Dénéchaud, Suzanne Duquet, Marian Scott, Agnès Lefort, Claire Fauteux, Paige Pinneo, peintres, et Sylvia Daoust, sculpteure. 
45 Femmes peintres au Canada, du 22 mars au 30 mars 1948, Eaton's Fine Art Gallery à Montréal.

46 "Canadian Women Painters ", du 2 avril au 16 avril 1949 à Montréal. L'exposition compte les artistes suivantes: Emily Carr, Pegi Nicol, Prudence Heward, Lilias Torrance Newton, Marian Scott, Jeanne Rhéaume, Ghitta Caiserman, Marion Arounson, Mary Miller, Mabel Lockerby, Rita Mount, Paige Pinneo, Didi Reusch, Sarah Robertson, Mary Grey Robinson, Anne Savage, Ethel Seath, Jori Smith, Betty Sutherland, Fanny Wiselberg, Rose Wiselberg. Cette liste provient de l' article anonyme, "Women's Works on Exhibit", The Standard, 2 avril 1949.

47 Du 3 mai au 24 mai 1950, Archives du Musée des beaux-arts de Montréal, dossicr A:E414.

48 Selon les archives du Musée des beaux-arts de Montréal, il n'existe pas d'exposition consacrée annuellement aux femmes à la Art Association of Montreal au cours des années trente et quarantc.

49 P. Harris McLarren, "Women's Art Sociery. Aim to Encourage Painters in Studio ", Montreal Star, 8 mars 1966. La Women's Art Society décernait chaque année des prix et des bourses d'études qui profitèrent éventucllement à certaines artistes professionnelles. C'est le cas de Claire Fauteux, qui grâce à une bourse de la Women's Art Society se rendit à Paris, à la fin des années trente, afin d'étudier à l'Académie Julian avec Maurice Denis.

50 Dans une perspective plus large et considérant l'apport des femmes dans le champ artistique en général, la contribution de la Women's Art Sociery nous apparaît essentielle; cependant cet article ne vise que l'analyse d'une exposition de groupe rassemblant des artistes professionnelles.

51 Sur la scène montréalaise, par exemple, les expositions de groupe sont sporadiques. Mentionnons "L'exposition de peintures féminines ", tenue à la Eaton Art Gallery à Montréal en mars 1933, clle rassemblait, entre autres, Berthe Des Clayes, Marjorie Smith, Sarah Robertson et Mary Grant. Au sujet de cette exposition, voir la critique de Henri Girard, "Exposition de peinture féminine chez Eaton ", Le Canada, 1 mars 1933. Mentionnons également, l'" Exposition de femmes artistes", tenue en mai 1934 à la Galerie William Scott \& Son à Montréal. Soulignons, de plus, "Oil Paintings by L.T. Newton, Prudence Heward, Anne Savage, Ethel
Seath ", organisée cette fois par l'Art Association of Montreal, du 4 au 30 décembre 1944. Malheureusement, les Archives du Musée des beaux-arts de Montréal n'ont conservé à ce sujet qu'un minimum de documents, ne nous permettant pas de juger de l'intention sous-tendant une telle exposition. Néanmoins, il s'agissait d'une collection d'artistes reconnues individuellement par la critique et, comme le titre l'indique, la réunion des femmes artistes semblait tout à fait gratuite. Voir, les Archives du Musée des beauxarts de Montréal, dossier E 258.

52 Kate Aitken, art. cit.

53 Pearl McCarthy, art. cit.

54 Anne Francis, art. cit.

55 Ibid.

56 Anonyme, Montreal Daily Star, art. cit.

57 Anonyme, La Patrie, art. cit.

58 Dorothy Farr et Nathalie Luckyj, From Women's Eyes, Women Painters in Canada, Kingston, Agnes Etherington Art Center, Queen's Universicy, 1976.

59 Marcel Clément, La femme dans la société, Trois-Rivières, Éditions du bien-public, 1953, p. 80.

60 Bien entendu, cettc attitude n'est pas exclusive aux milieux artistiques canadiens. Voir, Rozsika Parker, The Subversive Stitch. Embroidery and the Making of the Feminine, London, The Women's Press, 1984.

61 Charles Doyon, Le Clairon, Saint-Hyacinthe, 22 avril 1949. Cet article était une critique de l'exposition "Canadian Women Painters", tenue à la West End Gallery, en 1949.

62 René Normand, Le Canada, 3 avril 1948.

63 Henri Girard, Le Canada, 1 mars 1933.

64 Charles Doyon, art. cit.

65 Charles Doyon, art. cit.: "De toutes celles-ci, Emily Carr est sans doute la plus généralement connue au pays. La plupart de ses oeuvres vigoureusement brossées furent exécutées dans la Colombie canadienne, dont les coutumes et les vestiges de l'art indien, lui ont fourni l'occasion d'écrire des livres intéressants."

66 René Normand, art. cit.

67 Ibid. 